\title{
Cultura gerencial del deporte en Ecuador: Una mirada desde los actores sociales
}

\section{Management culture of sport in Ecuador: A look from social actors}

INFORMACIÓN DEL ARTÍCULO

Fecha de recepción: 17 de Septiembre de 2018.

Fecha de aceptación: 10 de Diciembre de 2018 .

\footnotetext{
${ }^{l}$ Doctor en Gerencia y Magíster en Gerencia Empresarial, Universidad Central de Venezuela. Docente de la Universidad Iberoamericana del Ecuador. E-mail: summar.gomez@gmail.com Código ORCID:

https://orcid.org/0000-0002-8132-5557
}

CITACIÓN: Gómez Barrios, S. A (2018). Cultura gerencial del deporte en Ecuador: Una mirada desde los actores sociales. Podium, 34, 35-56. doi:10.31095/podium.2018.34.3

ENLACE DOI:

http://dx.doi.org/10.31095/podium.201 8.34 .3

Summar Alfredo Gómez Barrios ${ }^{1}$

\section{Resumen}

Este artículo tiene por objeto analizar la cultura gerencial del deporte en Ecuador, haciendo énfasis en el alto rendimiento y en las ligas barriales. La investigación implicó la realización de entrevistas en profundidad a personas clave que ejercen cargos gerenciales en estos segmentos deportivos, razón por la cual la metodología se apoyó en un enfoque cualitativo, descriptivo y de campo, materializado en el método de la teoría fundamentada. Los resultados revelaron que existe un quehacer gerencial que en gran medida se ejerce sin las necesarias competencias profesionales, técnicas y humanas, con debilidades en la formación académica y con escasa capacitación en el área gerencial. Se concluye que estos factores adecuadamente alineados con acciones efectivas y bajo un destino compartido garantizarían un desempeño exitoso, a los fines de encaminar al deporte ecuatoriano a mejores resultados en el ámbito local, regional e internacional.

\section{Palabras Clave:}

Gerencia; cultura gerencial; organizaciones del deporte; deporte de alto rendimiento; ligas barriales; teoría fundamentada.

Clasificación JEL: M14.

\begin{abstract}
The purpose of this article is to analyze the managerial culture of sport in Ecuador, emphasizing high performance and neighborhood leagues. The research involved conducting in-depth interviews with key people who hold management positions in these sports segments, which is why the methodology was based on a qualitative, descriptive and field approach, materialized in the method of grounded theory. The results revealed that there is a managerial task that to a large extent is exercised without the necessary professional, technical and human skills, with weaknesses in academic training and with little training in the managerial area. It is concluded that these factors, properly aligned with effective actions and under a shared destiny, would guarantee a successful performance, in order to direct the Ecuadorian sport to better results at the local, regional and international level.
\end{abstract}

Keywords:

Management; managerial culture; sports organizations; High intensity sport; neighborhood leagues, grounded theory.

JEL Classification: M14. 


\section{Introducción}

El deporte es una actividad reconocida universalmente y está muy bien organizada en todos los países, el cual es practicado por un alto porcentaje de la humanidad y es concebido como arte, ciencia, espectáculo y salud. En Ecuador el deporte se encuentra arraigado en la sociedad y en los últimos años ha alcanzado un posicionamiento significativo. Se observa en la vida cotidiana y a través de los medios de comunicación social cómo aparece de manera recurrente información vinculada a esta actividad, convirtiéndose en un eje clave que abarca lo socioeconómico, lo político y lo axiológico, teniendo un interés manifiesto en sectores donde anteriormente carecía de importancia. Hoy día, el Estado ecuatoriano, así como las gobernaciones, alcaldías, instituciones públicas y privadas se apoyan en el deporte y en sus figuras deportivas como íconos referenciales de éxito para destacar sus resultados en la gestión. Son aspectos que sin duda otorgan relevancia al deporte, especialmente al alto rendimiento $\mathrm{y}$ al barrial, dos contextos deportivos centrados en la cultura gerencial como fenómeno de interés en esta investigación.

Sobre la base de las consideraciones anteriores, es pertinente hacer una aproximación conceptual en ambos segmentos deportivos. En este tenor el deporte barrial, también denominado deporte para todos, popular o de masas, hace referencia a aquellas actividades físicas que se realizan de manera libre y espontánea con propósitos netamente lúdicos, cuya práctica favorece a grandes masas, que además está sustentado en una tradición cultural (Arias, 2017). Esta definición acerca del deporte barrial se complementa con la siguiente:

... está orientado a favorecer a grandes sectores de la sociedad, con el propósito de elevar el bienestar social y la calidad de vida a través de actividades físico-deportivas, atendiendo aspectos vinculados al mejoramiento de la salud corporal, el sosiego emocional, la satisfacción personal, y por ende evitar el sedentarismo, propiciando actitudes positivas en las personas (Aguilar, 2014, p. 54).

Es evidente entonces, que el deporte barrial está encaminado a ofrecer notables beneficios a grandes sectores de la sociedad, fundamentalmente en mejorar la calidad de vida, el buen uso del tiempo libre, la inclusión social, además de la satisfacción mental, física y emocional. En sí, el deporte barrial o de masas está ubicado en la base de la pirámide deportiva, y en su parte más alta está el deporte de alto rendimiento (Cazorla, 1979). Por su parte, el deporte de alto rendimiento es entendido como aquel que se caracteriza por realizarse de forma recurrente, sostenida y sistemática con elevados niveles de exigencia y centrado en escenarios internacionales altamente competitivos (Arias, 2017). Visto así, es una subcategoría de actividad física, altamente especializada y de carácter competitivo realizada en forma individual o colectiva, subordinada al acatamiento de reglas y normas institucionalizadas "...que requiere de entrenamiento físico y que generalmente se realiza a altas intensidades. De modo 
que su objetivo principal es una competencia que siempre arroja resultado" (Villegas, 2012, p. 12).

Ahora bien, aun cuando el deporte de alto rendimiento y el deporte barrial operan bajo diferentes perspectivas de acuerdo con su finalidad como se ha señalado, ambas coinciden en que son estructuras organizativas mediante las cuales se ordena y coordina el trabajo del grupo de personas que cumplen funciones, responsabilidades y tareas según su cargo y jerarquía para lograr los objetivos y metas programadas.

A tal efecto, una organización del deporte orientada bien sea al gran espectáculo competitivo o al deporte para todos sin distingo de sexo, edad, etnia, condición física o mental, no puede lograr aquello que se ha propuesto en términos de cantidad y calidad si las personas dentro de estas instituciones ejercen sus labores de forma individual; más bien deben funcionar bajo un sistema de relaciones alineadas con objetivos claros, con propósitos compartidos y con beneficios comunes, mediante la cual una organización del deporte se soporta en el alineamiento de acciones en conjunto de las personas, considerando sus responsabilidades dentro de los distintos niveles jerárquicos, a los fines de alcanzar los objetivos planteados (Aguilar, 2014).

En virtud de ello, las organizaciones del deporte requieren de un quehacer gerencial que precisamente pueda en su práctica, materializar el conjunto de acciones de las personas vinculadas a la unidad que conduce, a los fines de cumplir con los objetivos y metas planteadas. En efecto, Calabuig, Gómez y Liceras (2010) consideran que el gerente del deporte representa uno de los principales factores dinamizantes de una organización, y dentro de sus funciones están las de planificar, organizar, dirigir y controlar; postura que se armoniza con la de Peiró, Ramos y Martínez-Tur (1995), precisando que la gerencia del deporte envuelve un conjunto de responsabilidades y tareas que involucran a las funciones administrativas de planificación, organización, evaluación y control de actividades.

Según lo expresado por los precitados autores, el gerente del deporte emerge como aquel actor dentro de una organización deportiva que debe asegurar resultados de excelencia, en conformidad con ajustados procesos de planificación, organización, dirección y control. Una concepción más amplia que expone la importancia de la gerencia del deporte, es la siguiente:

El éxito de las operaciones depende del conocimiento que los gerentes tengan sobre factores internos, como los niveles de trabajo, las habilidades y capacidad de los empleados, las funciones y la motivación respecto a los objetivos de la organización, etc. De igual importancia es la percepción que tengan los gerentes respecto a factores externos como la economía mundial, la economía local, la tecnología, la política, la penetración del mismo mercado por otros competidores, las necesidades de los jóvenes y sus tendencias y el contexto social y cultural de la juventud (Acosta, 2005, p. 252). 
Es un tablero que refleja claramente la valoración que se otorga al gerente del deporte que posee el conocimiento y las capacidades indispensables para manejar con propiedad aquellos factores internos y externos que impactan a las organizaciones del deporte. En este tenor, se evidencian en los factores internos aspectos organizativos y de dirección, gran parte de ellos de características subjetivas y que conciernen a las capacidades tanto intrapersonales como interpersonales. En cuanto a los factores externos, se deduce por un lado la presencia del macroentorno, relativo a la esfera nacional e internacional en cuanto a lo político, económico, social, demográfico, entre otros, y por otro con el microentorno, que corresponde a las necesidades específicas que demandan los actores involucrados directamente en el deporte y aquellos grupos de interés.

Este escenario a todas luces obliga a un gerente del deporte a estar formado, con el fin último de operar con efectividad y bajo parámetros de excelencia. Al respecto, Vargas (2011) considera que un gerente del deporte debe tener preparación, formación $\mathrm{y}$ capacitación en competencias de dirección para interactuar con éxito en el sistema deportivo. A su turno, Campos (2007) sostiene que el deporte necesita de gerentes formados y titulados de la actividad física y del deporte, y dentro de estos, deben seleccionarse a los más calificados.

Desde esta perspectiva, se coincide que un gerente del deporte debe caracterizarse por adquirir y desarrollar conocimientos y competencias deseables para el ejercicio del rol, los cuales se logran indefectiblemente con la formación académica y a través de procesos permanentes de capacitación. Adicionalmente, Aguilar y Guerra (2015) indican que además del conocimiento y las competencias del gerente del deporte, se debe agregar la experiencia lograda mediante su práctica en escenarios deportivos, aspecto que ayudaría a tener un conocimiento anticipado de las circunstancias que rodean a este sector. Así pues, conocimiento, competencias y experiencia son tres aspectos que se convierten en factores críticos de éxito para la acción gerencial, dado que de este actor se depende en gran medida para que una organización del deporte pueda ser exitosa.

Visto este panorama, el gerente del deporte tiene un rol esencial dentro del sistema deportivo bien sea desde la perspectiva del alto rendimiento o desde el deporte barrial. Una actuación gerencial deseable dadas las características expuestas, cuya permanencia en el tiempo dependerá del establecimiento de una sólida cultura sustentada en aquellos aspectos subjetivos que están presentes en el ser humano y que encierran componentes axiológicos, actitudinales y emocionales, los cuales se conectan a través de un sistema de símbolos culturales que se reflejan a través del comportamiento y de las relaciones de las personas dentro de las organizaciones del deporte. Estos sistemas simbólicos de la cultura se complementan de la siguiente manera: 
Los sistemas simbólicos de la cultura (...) se dan a través de códigos (ajustados a un lenguaje característico y un comportamiento social entre pares, colaboradores y relacionados) como las percepciones, emociones, actitudes, hábitos, creencias, valores, tradiciones, rutinas y otras formas de interacción dentro y entre estos grupos existentes en estas organizaciones, sistemas simbólicos fijados por un conjunto de reglas y normas que deben cumplirse (Gómez, 2017, p. 122).

Según se observa, el comportamiento de los actores sociales y el lenguaje son códigos que se relacionan entre sí y en su esencia responden a un sistema de símbolos culturales (Geertz, 1987; Schneider, 1980). Visto así, la cultura es la forma como un grupo de personas organizan sus experiencias en torno a una realidad concreta a través de sus percepciones, comprendiendo e interpretando las interacciones cotidianas que orientan el comportamiento de sus miembros (Levy-Strauss, 1985; Goodenough, 1981). Sobre la base de estas consideraciones, no hay dudas que el denominador común en una cultura es el uso de un sistema de símbolos, un lenguaje específico y unas reglas que regulan las relaciones.

Resulta evidente entonces que la cultura es un marco de referencia compartido y aceptado por el grupo de personas de una organización, orientando la manera de pensar y actuar frente a las circunstancias, donde se incluyen aspectos intangibles (actitudes, valores, emociones) y tangibles vinculados a las operaciones cotidianas; en sí, una cultura organizacional que se configura como un sistema mediante el cual interactúan personas y que significa “... un modo de vida, un sistema de creencias y valores, una forma aceptada de interacción y de relaciones típicas y normas que comparten los miembros de determinada organización" (Gómez, 2017, p. 180). En resumidas cuentas, la cultura organizacional envuelve y afecta todo cuanto ocurre en la organización.

Más aún, es una cultura organizacional que debe ser atendida y conducida por un quehacer gerencial que además de planificar, estructurar y sistematizar las complejidades que encierra la dinámica organizativa, tenga la capacidad para inspirar a otros (Kotter, 2007); todo ello con el propósito de garantizar la concordancia entre los planes y sus resultados. En sí, una cultura gerencial que ejerza su influencia para movilizar a las personas $y$ por consiguiente a las organizaciones del deporte hacia una visión compartida.

En este orden de ideas, Gómez (2017) revela que las organizaciones del deporte que posean una firme cultura gerencial, están en la capacidad de integrar aspectos como los hábitos, rutinas y modos de conducta aprendidos en la vida organizacional, así como la valoración hacia el trabajo, el acoplamiento de los distintos roles que garantizan la cohesión grupal, la creación de ajustados procesos de comunicación por razones técnicas o por motivos afectivos y emocionales, la instauración de un sistema de valores y creencias compartidas, entre otros aspectos.

Cabe agregar también, las aportaciones de Etking y Schvarstein 
(1992), quienes enfatizan que la cultura gerencial condiciona los modos de pensar, crecer, hacer y cumplir las funciones y tareas gerenciales dentro de una organización, donde están presentes las formas de interacción social comunicadas, difundidas, mantenidas y compartidas por los grupos, mediante un sistema que funciona con un lenguaje común, entendido por todos los actores sociales.

Correspondientemente, Granell (1997) advierte que la cultura gerencial está focalizada en la incubación de cambios y transformaciones que sean provechosas y pertinentes para el éxito organizativo, sin perder de vista aquellas transformaciones que se produzcan en la aldea global. Después de todo, se puede entender que la cultura gerencial pasa a ser un marco de referencia aceptado por los actores sociales inmersos en el deporte, reflejando un modo de pensar y actuar del actor-gerente frente a situaciones concretas, dando direccionalidad a las acciones.

En torno a los elementos señalados, la investigación estuvo focalizada en analizar la cultura gerencial del deporte en Ecuador, considerando dos grandes segmentos: el deporte de alto rendimiento y las ligas barriales. En este sentido, fueron abordados en este estudio actores clave con responsabilidades gerenciales en el deporte ecuatoriano vinculados con estos segmentos, con la finalidad de que pudieran dar cuenta de lo que acontece en la realidad empírica. En definitiva, cuál era su visión con respecto al fenómeno objeto de tratamiento y cómo son los patrones de comportamiento a partir de tres categorías previamente seleccionadas: (a) Perfil Gerencial; (b) Características de la Cultura Organizacional; y (c) Concepción de la Cultura Gerencial.

Este estudio se justifica, dada la insuficiencia de investigaciones en el sector deportivo ecuatoriano, principalmente en el campo de la gerencia, cuyos resultados servirán de plataforma para dar continuidad a proyectos investigativos de similar naturaleza, así como su contribución en la producción y ampliación de conocimientos, proporcionando aportes teóricos importantes y de valor utilitario al contexto social para que puedan ser replicados en otras organizaciones promotoras del deporte.

\section{Metodología}

Dado que la realidad es un sistema en permanente interacción y cambio, en este estudio se dispuso asumir el enfoque cualitativo, que a su vez condicionó el tipo de investigación en cuanto a nivel y diseño. El enfoque cualitativo tuvo la intención de analizar e interpretar la realidad desde la perspectiva de los actores sociales, a fin de comprender lo que sucede en el fenómeno objeto de estudio. En este contexto, intentar caracterizar el enfoque cualitativo requirió de sistematización, cuyo interés central fue interpretar el significado que le otorgan los propios actores a su vida social. Para ello, los principales aspectos que delimitan esta modalidad de investigación están: (a) el mundo social, 
el sujeto, la subjetividad y la cultura; (b) la actividad que realiza el actor; (c) los procesos en que interactúa; (d) los significados de la acción; (e) las definiciones subjetivas de la acción; y (f) el lenguaje y la comunicación (Rusque, 2007).

En efecto, este enfoque permitió construir el conocimiento mediante las interpretaciones que surgieron del interior de la consciencia subjetiva de los actores sociales. Bajo esta premisa, fue necesario adentrarse en el ambiente donde los sujetos consultados interactúan, con el objeto de recabar datos a partir de sus expectativas, vivencias y experiencias que suscriben sus comportamientos; datos que fueron registrados y utilizados como plataforma para realizar interpretaciones de la concepción que tienen acerca del fenómeno estudiado.

Al mismo tiempo, el estudio se ubicó en una investigación descriptiva mediante la cual se identificaron y analizaron en su justa dimensión aquellos aspectos atinentes al quehacer gerencial que determina la cultura prevaleciente en las organizaciones del deporte ecuatoriano seleccionadas en este estudio; tipo de investigación que permitió recabar datos de interés para ser conceptualizados, categorizados e interpretados desde una perspectiva cualitativa, a partir del significado que le otorgan las personas (Cerda, 1991).

Asimismo, el diseño estuvo focalizado en una investigación de campo para tener una relación directa con los actores sociales en su ambiente natural (Arias,
2016), a los efectos de lograr una ajustada interpretación de aquello que es vivido, sentido y percibido por ellos; en sí, su manera de ver y comprender su realidad, cuya interpretación cualitativa de los datos se trianguló con un análisis cuantitativo para fortalecer el estudio (Martínez, 2009).

Para tales propósitos, la investigación se sustentó en la teoría fundamentada (Strauss y Corbin, 2002), la cual permitió sistematizar los datos que se iban obteniendo de la realidad. Este método se soportó en cinco grandes factores: (1) El muestreo teórico; (2) El método comparativo constante; (3) La codificación abierta; (4) La codificación axial; y (5) La codificación selectiva.

En torno a lo anterior, la investigación se inició con el muestreo teórico, que consistió en seleccionar de manera intencional a un experto para ser entrevistado en profundidad, cuyo criterio fue que tuviese amplios conocimientos y experiencia en la gerencia del deporte, además de la vivencia en escenarios de competencia como atleta y/o entrenador, y ser profesional universitario.

Una vez realizada y transcrita la entrevista, se configuró como una unidad hermenéutica para ser analizada primeramente en codificación abierta a objeto de fragmentar los datos, identificar conceptos, etiquetarlos y clasificarlos en categorías, y luego en codificación axial para relacionar los conceptos emergentes con sus respectivas categorías a fin de determinar el tipo de relación. Culminado el análisis de la entrevista, se procedió a realizar nuevamente el muestro teórico 
con otro entrevistado bajo los mismos criterios de selección y el mismo procedimiento en codificación abierta y axial; muestreo teórico que continuó de manera reiterada con otros entrevistados y finalizó cuando hubo saturación teórica, vale decir, cuando no emergieron nuevos datos para ser procesados.

Cabe indicar que durante todo el proceso de análisis en ambas modalidades de codificación, se implementó el método comparativo constante dentro de una misma entrevista y entre ellas, de manera de depurar datos que garantizaran el adecuado ordenamiento conceptual en cuanto a conceptualización, categorización y relaciones entre sí.

Finalmente, los datos que emergieron en ambas codificaciones se integraron $y$ refinaron en codificación selectiva para realizar interpretaciones de los hallazgos encontrados en la realidad empírica; hallazgos que luego fueron contrastados con el estado del arte para determinar las brechas. En definitiva, la investigación implicó un total de cuatro actores entrevistados con el necesario potencial y relevancia como se muestra en la tabla 1.

\section{Resultados}

\section{Análisis en codificación abierta y codificación axial}

El proceso de análisis en codificación abierta requirió examinar e interpretar los datos de forma rigurosa y detallada. Debido ello, desde el inicio del análisis textual de los documentos primarios convertidos en unidades hermenéuticas,

\section{Tabla 1.}

Actores entrevistados en calidad de expertos con base en el conocimiento, la experiencia y la vivencia en el deporte.

\begin{tabular}{|c|c|c|}
\hline ENTREVISTADO & CARGO & RESUMEN DE SU EXPERIENCIA \\
\hline Jorge Granja Cobos & $\begin{array}{l}\text { Vicepresidente de la Federación } \\
\text { Ecuatoriana de Voleibol }\end{array}$ & $\begin{array}{l}\text { Ex atleta y entrenador de selección nacional. Licenciado } \\
\text { en Cultura Física del Instituto Lenin de Moscú. Docente } \\
\text { en la Universidad de San Francisco de Quito. Vinculado } \\
\text { al deporte por } 40 \text { años. }\end{array}$ \\
\hline José Antonio Guevara & $\begin{array}{l}\text { Vicepresidente de la Concentración } \\
\text { Deportiva de Pichincha. } \\
\text { Vicepresidente de la Federación Deportiva } \\
\text { Nacional del Ecuador }\end{array}$ & $\begin{array}{l}\text { Ex atleta. Licenciado en Administración Pública. } \\
\text { Docente de la Universidad Central de Ecuador. Ha sido } \\
\text { miembro de distintas organizaciones del deporte, entre } \\
\text { ellas Vicepresidente del Comité Olímpico Ecuatoriano. } \\
\text { Más de } 50 \text { años relacionado con el deporte. }\end{array}$ \\
\hline Hugo León & Presidente de la Liga Barrial de Quito & $\begin{array}{l}\text { Más de } 60 \text { años en el deporte. Ex atleta de alto } \\
\text { rendimiento y Profesor Normalista. Dirigente en distintas } \\
\text { organizaciones del deporte. }\end{array}$ \\
\hline Pedro Fernández & $\begin{array}{l}\text { Secretario de Educación, Deporte y } \\
\text { Cultura del Distrito Metropolitano de Quito }\end{array}$ & $\begin{array}{l}\text { Abogado con un master en negocios y administración } \\
\text { de fútbol en el Johan Cruyff Institute. Experiencia en } \\
\text { marketing deportivo. Más de } 30 \text { años en el deporte y } \\
\text { actualmente sigue activo como atleta. }\end{array}$ \\
\hline
\end{tabular}

Fuente: Elaboración propia. 
fueron emergiendo etiquetas conceptuales producto de la fragmentación de estos datos; etiquetas que se clasificaron en tres categorías previamente seleccionadas en función de sus propiedades o atributos, como son:

- Perfil Gerencial: Rasgos asociados al ejercicio gerencial que aseguran comportamientos deseables para un desempeño exitoso.

- Características de la Cultura Organizacional: Atributos de un sistema determinado por el conjunto de relaciones interpersonales existentes en la organización en conexión con la filosofía organizacional.

- Concepción de la Cultura Gerencial: Postura que se tiene respecto a la manera de ver y concebir el quehacer gerencial desde la perspectiva de una cultura orientada a generar cambios personales, grupales y organizacionales.

Esta fragmentación de datos facilitó el ordenamiento conceptual en codificación abierta, que al consolidarlos se generó un

Tabla 2

Ordenamiento conceptual en codificación abierta.

\begin{tabular}{cccc}
\hline \multirow{2}{*}{$\begin{array}{c}\text { UNIDADES } \\
\text { DE ANÁLISIS }\end{array}$} & $\begin{array}{c}\text { Cerfil } \\
\text { Gerencial }\end{array}$ & $\begin{array}{c}\text { Características de la } \\
\text { Cultura Organizacional }\end{array}$ & $\begin{array}{c}\text { Concepción de la } \\
\text { Cultura Gerencial }\end{array}$ \\
\cline { 2 - 4 } & 13 & 17 & 16 \\
\hline Entrevistado 1 & 6 & 9 & 9 \\
\hline Entrevistado 2 & 7 & 12 & 14 \\
\hline Entrevistado 3 & 5 & 22 & 56 \\
\hline Entrevistado 4 & 31 & 60 & $38,10 \%$ \\
\hline TOTAL & $21,09 \%$ & $40,81 \%$ &
\end{tabular}

Fuente: Elaboración propia. total de 147 etiquetas conceptuales agrupadas en las tres categorías aludidas (Tabla 2).

En este sentido, las cifras indican que el $40,81 \%$ de las etiquetas conceptuales fueron clasificadas en la categoría "Características de la cultura organizacional", muy cercana a la categoría "Concepción de la cultura gerencial" con $38,10 \%$, datos que se reflejaron de manera parecida en cada una de las unidades de análisis.

Estos datos dan cuenta del interés por prevalecer aquellos aspectos referidos a la filosofía organizacional, vale decir cuál es la razón de ser de las organizaciones, cómo se proyectan de cara al futuro y cómo se guía el comportamiento de las personas, en sí el interés en la misión, la visión y los valores organizativos, además de las creencias, las rutinas, los hábitos, entre otros; filosofía organizacional que de igual modo necesita como condición para su cumplimiento de un quehacer gerencial que combine la autoridad y el poder formal que se otorga para planificar,

Fuente: Elaboracion propia. 
estructurar y sistematizar las complejidades que envuelven la dinámica de una organización con la capacidad para influenciar, persuadir e inspirar a otros. Conforme a esto, una cultura organizacional y gerencial que contribuya a reorientar comportamientos conducentes al logro de los objetivos estratégicos y metas en las organizaciones del deporte ecuatoriano.

Por otra parte, las 147 etiquetas conceptuales que emergieron en codificación abierta fueron analizadas en codificación axial a los efectos de relacionarlas con sus respectivas categorías y determinar el tipo de relación con base en las siguientes prescripciones:

- Es una Propiedad: Rasgos que identifican y se diferencian del resto de los tipos de relación, caracterizando la esencia de un concepto dentro de una categoría.

- Es una Consecuencia: Aquel concepto que se produce como resultado de una acción o hecho derivado de una categoría.

- Es parte: Aun cuando no es un rasgo que lo distingue, es un componente o segmento de un concepto con respecto a la categoría.

- Es contradictoria: Conceptos que discrepan con respecto a la categoría.

Al enlazar estos tipos de relación entre las etiquetas conceptuales y las categorías "Perfil gerencial", "Características de la cultura organizacional" y "Concepción de la cultura gerencial", la tabla 3 muestra

Tabla 3.

Relación de etiquetas conceptuales con categorías en codificación axial.

\begin{tabular}{|c|c|c|c|c|c|c|}
\hline & \multirow[b]{2}{*}{ CATEGORÍAS } & \multicolumn{4}{|c|}{ TIPOS DE RELACIÓN DE ETIQUETAS CONCEPTUALES } & \multirow[b]{2}{*}{ TOTAL } \\
\hline & & $\begin{array}{c}\text { Es una } \\
\text { Propiedad }\end{array}$ & $\begin{array}{c}\text { Es una } \\
\text { Consecuencia } \\
\end{array}$ & $\begin{array}{c}\text { Es } \\
\text { Parte } \\
\end{array}$ & $\begin{array}{c}\text { Es } \\
\text { Contradictoria } \\
\end{array}$ & \\
\hline \multirow{5}{*}{ Entrevistado 1} & Perfil Gerencial & 4 & 1 & 5 & 3 & 13 \\
\hline & Características de la Cultura Organizacional & 4 & 1 & 3 & 9 & 17 \\
\hline & Concepción de la Cultura Organizacional & 6 & 2 & 4 & 4 & 16 \\
\hline & TOTAL & 14 & 4 & 12 & 16 & 46 \\
\hline & $\%$ & $30,43 \%$ & $8,70 \%$ & $26,09 \%$ & $34,78 \%$ & $100 \%$ \\
\hline \multirow{5}{*}{ Entrevistado 2} & Perfil Gerencial & 1 & 0 & 1 & $\overline{4}$ & 6 \\
\hline & Características de la Cultura Organizacional & 2 & 0 & 2 & 5 & 9 \\
\hline & Concepción de la Cultura Organizacional & 4 & 0 & 0 & 5 & 9 \\
\hline & TOTAL & 7 & $\mathbf{0}$ & 3 & 14 & 24 \\
\hline & $\%$ & $29,17 \%$ & $0,00 \%$ & $12,50 \%$ & $58,33 \%$ & $100 \%$ \\
\hline \multirow{5}{*}{ Entrevistado 3} & Perfil Gerencial & 3 & 1 & 1 & 2 & 7 \\
\hline & Características de la Cultura Organizacional & 3 & 1 & 0 & 8 & 12 \\
\hline & Concepción de la Cultura Organizacional & 9 & 1 & 1 & 3 & 14 \\
\hline & TOTAL & 15 & 3 & 2 & 13 & 33 \\
\hline & $\%$ & $45,46 \%$ & $9,09 \%$ & $6,06 \%$ & $39,39 \%$ & $100 \%$ \\
\hline \multirow{5}{*}{ Entrevistado 4} & Perfil Gerencial & 3 & 1 & 1 & 0 & 5 \\
\hline & Características de la Cultura Organizacional & 3 & 6 & 1 & 12 & 22 \\
\hline & Concepción de la Cultura Organizacional & 4 & 2 & 2 & 9 & 17 \\
\hline & TOTAL & 10 & 9 & 4 & 21 & 44 \\
\hline & $\%$ & $22,72 \%$ & $20,46 \%$ & $9,10 \%$ & $47,72 \%$ & $100 \%$ \\
\hline \multirow{5}{*}{$\begin{array}{c}\text { Total } \\
\text { Entrevistados }\end{array}$} & Perfil Gerencial & 11 & 3 & 8 & 9 & 31 \\
\hline & Características de la Cultura Organizacional & 12 & 8 & 6 & 34 & 60 \\
\hline & Concepción de la Cultura Organizacional & 23 & 5 & 7 & 21 & 56 \\
\hline & TOTAL & 46 & 16 & 21 & 64 & 147 \\
\hline & $\%$ & $31,29 \%$ & $10,89 \%$ & $14,29 \%$ & $43,53 \%$ & $100 \%$ \\
\hline
\end{tabular}

Fuente: Elaboración propia. 
cifras que evidencian las tendencias tanto en el total de entrevistados como en cada uno de éstos, dispuestos como unidades de análisis.

En este tenor, los resultados arrojados en el consolidado de datos de cada una de las entrevistas realizadas a expertos y en el consolidado de datos del total de estas entrevistas, se puede apreciar una inclinación hacia el tipo de relación "Es contradictoria", con un pico de 58,33\% en el entrevistado 2. De igual forma, en el entrevistado 1, en el entrevistado 4 y en el total de entrevistados tuvieron $34,78 \%$, $47,72 \%$ y $43,53 \%$ respectivamente en este tipo de relación. Solamente en el entrevistado 3 destacó otro tipo de relación: "Es una propiedad" con 45,46\%, pero seguido de "Es contradictoria" con $39,39 \%$.

Estos datos permiten inferir que aun cuando se tiene el deseo de un ejercicio gerencial cuyo perfil debiera estar condicionado por un actor-gerente con las competencias requeridas, matizadas por los conocimientos y las capacidades logradas en las universidades, combinadas a su vez con la experiencia y vivencia en el deporte, la percepción que tienen estos actores de la realidad es más bien un comportamiento gerencial poco cónsono en cuanto al conocimiento y las habilidades que deberían poseer, dado que se adolece de formación universitaria para poder conducir las acciones del conjunto de personas adscritas a las organizaciones del deporte en el interés de responder a las necesidades que demanda el sector deportivo en Ecuador. En definitiva, no es suficiente tener la experiencia en campo para dirigir las complejidades que encierra la esfera deportiva, criterio que ha prevalecido en su escogencia.

\section{Consideraciones acerca de los resultados}

Después de finalizado el proceso de análisis de los datos en codificación abierta y axial de las entrevistas a expertos, donde se logró identificar conceptos, que a su vez fueron etiquetados y clasificados en categorías, y consecutivamente relacionados entre sí para determinar el tipo de relación, se procedió a implementar el análisis en codificación selectiva, donde se integraron los datos en cada una de las categorías seleccionadas en este estudio, para: (A) Realizar la interpretación de los hallazgos encontrados en el proceso investigativo con base en las tres categorías seleccionadas, bajo dos vertientes: La primera, la visión que tenían los actores, y la segunda, el comportamiento que opera en la realidad; y (B) Contrastar el estado del arte con los hallazgos encontrados.

\section{A) Interpretación de los hallazgos encontrados}

\section{Perfil gerencial}

Con respecto a la visión de los actores en esta categoría, se detecta que para ejercer funciones gerenciales en las organizaciones del deporte, su perfil debe tener como condición una acumulada experiencia en este sector, al igual que conocimientos del deporte a gerenciar, a 
fin de tener una clara perspectiva de sus necesidades y poder anticiparse a los constantes cambios que se producen en la esfera nacional e internacional. De igual modo, se aspira que el candidato sea profesional universitario y que a su vez posea herramientas gerenciales para maniobrar con propiedad los procesos administrativos; habilidades de liderazgo para influenciar y coordinar esfuerzos con proyección a futuro; y mística deportiva para alcanzar altos niveles competitivos. Adicionalmente, es deseable que un gerente debiera de antemano haber practicado algún deporte; tener relaciones con las federaciones y demás estamentos del deporte; conocer las leyes ecuatorianas, en especial la Ley del Deporte; y poseer ciertos conocimientos sobre marketing deportivo.

En cuanto al comportamiento que opera en la realidad, se revela que existen discrepancias entre la visión de los actores y cómo se ejerce la acción gerencial. En efecto, se sostiene que es práctica común el ejercicio de una gerencia basada en la experiencia que se tiene en el deporte sin la formación requerida para el cargo, además que buena parte de la dirigencia ecuatoriana no posee nivel universitario, acarreando un limitado conocimiento en materia gerencial y sin una formación real sobre los procesos administrativos que orienten la conducción idónea de los destinos del deporte, con el agravante que existe en estos cargos personas con un bajo nivel cultural que se traduce en un manejo inadecuado de las relaciones ente pares y colaboradores. Como corolario, se adolece de escuelas de formación en gerencia del deporte. En definitiva, son carencias que en gran medida impiden alcanzar logros importantes y resultados competitivos en escenarios internacionales.

Cabe acotar la afirmación que hace uno de los entrevistados "cuando no existía desarrollo deportivo ¡Quizás era factible un gerente del deporte sin formación!"; afirmación que resulta pertinente, dado los avances en cuanto a funcionamiento, productividad y formas organizativas para gestionar el deporte fundamentado en el conocimiento, razón por la cual es conveniente una gerencia del deporte altamente calificada y centrada en garantizar resultados acordes con los grandes retos que demanda el deporte ecuatoriano.

\section{Características de la cultura organizacional}

Como producto de los datos obtenidos de las respuestas de los entrevistados referidos a la visión que tienen sobre esta categoría que estiman deseable para desarrollarse y crecer con éxito, se constata que a las organizaciones del deporte le correspondería involucrar a todos sus miembros en el ánimo de que conozcan en profundidad su filosofía, la cual apunta a la misión, la visión y los valores organizativos, y que a su vez puedan realizar aportes a la dinámica que rodea los procesos, tareas y acciones a seguir alineados con esta filosofía. Estos aspectos en su esencia contribuirían a una mayor identificación institucional, aspectos que resultan muy atinentes con respecto al testimonio de un entrevistado "que se pongan la camiseta, eso falta en 
muchas organizaciones deportivas". Es evidente entonces que las organizaciones del deporte aspiran una cultura que implique la integración, cohesión, sentido de pertenencia y compromiso ético entre sus miembros y con la organización; una cultura organizacional cuya tendencia esté orientada hacia la práctica de una gestión mucho más profesional, aunando esfuerzos en conjunto y colocando el foco en los resultados que se desean alcanzar.

En relación con el comportamiento que prevalece en las organizaciones del deporte en Ecuador, se confirma que se tiene consolidada una estructura organizativa que parte desde el Comité Olímpico Ecuatoriano y aguas abajo se articula con las federaciones, las ligas, los clubes y los equipos. Asimismo, se valora como parte sustancial de esta estructura, la creación del Ministerio del Deporte, una excelente iniciativa mediante la cual se ha invertido en infraestructura deportiva y se ha instaurado centros de alto rendimiento; aun así, se considera que dicha infraestructura no está a la altura de otros países y no se tiene una adecuada preservación y mantenimiento.

Además de lo anterior, este organismo ministerial todavía debe consolidar ciertos procesos como la necesaria atención al deportista élite, no solamente en el aspecto económico que de alguna manera ayuda a paliar necesidades personales para que pueda dedicarse a su preparación, sino también en recibir el primordial acompañamiento de un equipo multidisciplinario vinculado a las ciencias aplicadas al deporte y de entrenadores formados $y$ altamente calificados.

A tal efecto, es conveniente la activación de procesos de capacitación de forma sistemática y permanente en el personal con funciones primarias y de apoyo hacia el atleta; por consiguiente, esta capacitación debe permear todos los niveles jerárquicos de su estructura organizativa, debiéndose complementar con la búsqueda de acuerdos con universidades como por ejemplo la Universidad Tecnológica Equinoccial, institución que tiene el interés en implantar estudios de tercer y cuarto nivel en Administración Deportiva, y la Universidad de las Américas que tiene opciones de estudios en Administración y Marketing Deportivo.

En las mismas circunstancias anteriores, se enfatiza que una importante proporción de los aportes económicos que realiza el Estado ecuatoriano están dirigidos al alto rendimiento, aportes que por demás debieran justificarse con resultados competitivos en el ámbito internacional producto de una planificación con base en varios ciclos olímpicos, como lo hacen las naciones de clases mundial y aquellos países que han tenido progresos importantes en la región. A pesar de ello, los procesos de planificación deportiva ecuatoriana en gran parte no cumplen con esta forma de concebir el futuro, una planificación que abarque dos o tres ciclos olímpicos para estimar hacia dónde se quiere llegar, que incluya un calendario competitivo promovido por el Estado, instando a la gerencia del deporte a que proyecte sus acciones a largo plazo y analizando en 
conjunto (autoridades deportivas y entes federativos) las expectativas que se desean cumplir. En cualquier caso, es justo reconocer que existen federaciones que están planificando con base en dos ciclos olímpicos y preparándose para las olimpiadas de 2024.

Estas demandas se enfrentan con algunas dificultades, en el hecho que precisamente las autoridades deportivas exigen resultados sin pasar por el tamiz de una evaluación previa, no funciona con planes estratégicos para conducir los objetivos y metas que deban plantearse, $y$ adicionalmente no tienen asesores con pleno conocimiento y experiencia en el deporte, matizados por la presencia de espacios de poder político en distintas instancias, incidiendo de manera importante en las decisiones que se toman; circunstancias que atentan contra el necesario respaldo para reconducir acciones que permitan el posicionamiento deportivo a escala internacional. En este mismo sentido, se advierte que no se valora al atleta élite que luego de cumplir años de preparación y participando con éxito en competencias internacionales, al llegar el momento de su retiro no se le presta el apoyo requerido, y en el mejor de los casos se dispensa una atención muy precaria que no ayuda a tener una mejor calidad en el trascurrir de vida.

Por otra parte, desde la perspectiva de los expertos entrevistados se corrobora que las ligas barriales atienden el $40 \%$ de los deportistas ecuatorianos cuyo foco central es mejorar los índices de sedentarismo en la población, incentivando hábitos saludables a través del deporte y la recreación, donde entes gubernamentales han realizado una loable labor con la creación de centros deportivos para atender a este sector, basándose en un modelo de gestión que asegure la preservación de canchas sintéticas con base en la implementación de mecanismos que orienten su adecuado uso, como por ejemplo la fijación de una cuota que favorezca su mantenimiento, eximiendo a aquellos sectores más vulnerables como discapacitados, niños, niñas y mujeres; aspectos que sin duda ayudan a crear conciencia en la gente, todo ello en el interés de que puedan apropiarse de estos espacios. Sin embargo, se aprecia que existen ligas que se encuentran bien dotadas y otras no tanto, producto de políticas deportivas hacia este sector que no están claras, que operan con poco personal y con aportes económicos escasos (en ocasiones con largos periodos sin recibirlos), motivo por el cual se han visto en la obligación de realizar algunas actividades para cancelar deudas que necesariamente han adquirido.

En este mismo orden de ideas, se revela que el deporte barrial no es una prioridad para el Ministerio del Deporte, más bien se ha centrado exclusivamente en el deporte de alto rendimiento (apreciación que discrepa de otros actores, quienes consideran que se trabaja para el buen vivir en menoscabo del alto rendimiento) y es atendido cuando los políticos están en campaña, a sabiendas que es un nicho que potencialmente genera votos para elegir alcaldes. Se acentúa también que muchos atletas que llegan al alto rendimiento provienen de 
las ligas barriales, siendo formados con escasos recursos y sus necesidades no son atendidas con efectividad por el Estado "No saben que el atleta sale de aquí ¡De las canchas de tierras!, como Morejón que salió de las canchas de nosotros en pistas de tierras, sin zapatos y esperan que sea campeona mundial para recién reconocerla" manifestaba un entrevistado.

\section{Concepción de la cultura gerencial}

En consideración a la interpretación de los datos obtenidos de las respuestas emitidas por los entrevistados expertos en torno a la visión que tienen acerca de la concepción de la cultura gerencial, se deduce que un gerente tiene la obligación de formarse para poder conducir con eficiencia y eficacia las responsabilidades inherentes al cargo. Por supuesto, para ejercer acciones gerenciales, es imperativo que un gerente evalúe permanentemente los procesos básicos de la administración, vale decir, una minuciosa revisión de la planificación, la organización, la dirección y el control de la gestión deportiva. Ello implica saber operar dentro de una estructura organizativa que asegure su solidez, con el liderazgo necesario para influenciar, persuadir y motivar a las personas a través de ajustados procesos de comunicación que tiendan a lograr compromiso en las acciones a seguir y que a su vez deriven en resultados cónsonos con los planes y proyectos en marcha.

Asimismo, un gerente debe ejercer sus funciones, responsabilidades y tareas tanto en la oficina como en el campo, para tener una percepción cercana de la realidad que dé cuenta de lo que allí sucede y pueda estar preparado para enfrentar los retos que se presenten, conociendo de primera mano aquellas inquietudes y necesidades del entorno deportivo como atletas, entrenadores, dirigentes, hinchada y demás grupos de interés por el deporte, sin que ello signifique que no delegue funciones en sus colaboradores para poder contrastar opiniones y considerar distintas alternativas que faciliten la toma de mejores decisiones.

En lo atinente a cómo se concibe la cultura gerencial del deporte en Ecuador sobre la base del comportamiento que opera en estas instituciones, se reafirma que el actor-gerente con formación académica garantizaría en buena parte resultados competitivos. Es este aspecto se reconoce que hay gerentes que poseen esta formación, algunos con nivel de maestría; aun así, no se tiene el suficiente personal con educación universitaria y capacitación en materia gerencial. Debido a ello, la práctica gerencial que hasta ahora ha prevalecido se refleja en la carencia de un pensamiento estratégico para planificar y que oriente las acciones futuras de las personas adscritas a las organizaciones del deporte para cumplir con los objetivos y metas que han sido planificadas.

Es un comportamiento gerencial que se circunscribe a cumplir con un Plan Operativo Anual por exigencias presupuestarias del Ministerio del Deporte, ente administrativo que no funciona con planes estratégicos, y según 
el criterio de los entrevistados no son los más capacitados, mostrando poco conocimiento sobre el deporte; una alta dirigencia que en sí no tiene una clara visión deportiva. En este sentido, si no hay direccionalidad para controlar la gestión de un Ministerio, menos aún en las demás esferas de la estructura deportiva.

A pesar de todo lo anterior, se constata que algunas federaciones han entendido la importancia de patrocinar sus eventos. A tal efecto, han asumido iniciativas para establecer convenios con empresas que permiten fortalecer la organización de eventos. No obstante, en líneas generales se adolece de gerentes con las herramientas necesarias para establecer relaciones que garanticen el patrocinio, $y$ tampoco hay iniciativas para desarrollar el marketing deportivo. Varias de estas herramientas gerenciales que se aluden están vinculadas a competencias blandas basadas en las actitudes, como la perseverancia para lograr los objetivos; la persistencia para superar dificultades; la responsabilidad por el desempeño personal; la disposición para relacionarse, detectar oportunidades, negociar y resolver desacuerdos, entre otras. Ciertamente, se entiende que existe mucho recelo de las empresas para invertir en el deporte porque perciben que no genera rentabilidad; de allí el desafío que tienen los gerentes para construir alianzas o convenios de cooperación con este sector.

Se resalta asimismo, la conveniencia de observar las mejores prácticas gerenciales en equipos deportivos de clase mundial y de aquellos países de la región como Perú, Venezuela y Colombia, que aventajan a Ecuador en el tema gerencial y que operan con excelentes profesionales con base en su formación académica de tercer y cuarto nivel, además de su comprobada experiencia, obteniendo resultados significativos a escala internacional.

En lo que respecta a las ligas barriales, se asevera que éstas funcionan con un personal gerencial con formación académica como doctores, abogados, administradores, médicos, inclusive ex asambleístas, aunque se confiesa que otros no tienen formación pero han tenido una ejemplar actuación dentro de esta liga, como lo ilustra un entrevistado "José Francisco Cevallos no tiene título universitario y fue uno de los mejores ministros, y ahora es gobernador. Hay que tener algo dentro para llevar a un equipo como Barcelona a donde está". Es una liga que ha crecido en número de provincias con esfuerzo y autogestión y a pesar de los recortes económicos se sigue trabajando con ahínco.

Es una modalidad de organización la cual entiende que gerenciar el deporte es una tarea ardua y retadora; un esfuerzo que necesariamente tiene que emplear procesos de autogestión para poder funcionar $y$ atender a la inmensa población de atletas que convergen en el deporte barrial. Procesos de autogestión que han permitido adquirir bienes en beneficio de esta colectividad.

B) Contraste del estado del arte y los hallazgos encontrados

A los efectos de determinar la brecha 
entre el estado del arte y los hallazgos encontrados en la investigación, se estimó en este último considerar aquellos que operan en la realidad empírica, vale decir los patrones de comportamiento que se derivan de la práctica gerencial del deporte de alto rendimiento y de las ligas barriales. Para tales propósitos, a continuación se presentan 11 aportes de 16 autores que permitieron contrastar teorías existentes con la manera como se concibió el fenómeno en la realidad en términos de coincidencias $y$ contradicciones en cada una de las categorías seleccionadas en este estudio.

En relación con la categoría Perfil Gerencial, al contrastar los aportes teóricos con las evidencias empíricas que emergieron en la investigación (Tabla 4), se pudo apreciar un comportamiento gerencial que se muestra contradictorio con respecto a los aportes "Procesos administrativos", "Capacidades interpersonales" y "Formación y capacitación". Son inconsistencias en el ejercicio del rol, dadas las carencias de formación académica y de procesos sistemáticos de capacitación en el área gerencial para el desarrollo de saberes, para la adquisición de habilidades interpersonales y para generar valor utilitario al deporte de alto rendimiento y barrial; carencias que a fin de cuentas no contribuyen a elevar el desempeño profesional.

A pesar de ello, se constató que buena parte de los gerentes en estos segmentos provienen precisamente del deporte, coincidiendo con "Experiencia en el

Tabla 4.

Coincidencias y contradicciones en perfil gerencial.

\begin{tabular}{|c|c|c|c|c|}
\hline \multirow{2}{*}{ AUTORES } & \multirow{2}{*}{ APORTES } & \multirow{2}{*}{ ESTADO DEL ARTE } & \multicolumn{2}{|c|}{ REALIDAD EMPÍRICA } \\
\hline & & & COINCIDENCIAS & CONTRADICCIONES \\
\hline $\begin{array}{c}\text { Calabuig, Gómez y } \\
\text { Liceras (2010); Peiró, } \\
\text { Ramos y Martínez-Tur } \\
\text { (1995) }\end{array}$ & $\begin{array}{c}\text { PROCESOS } \\
\text { ADMINISTRATIVOS }\end{array}$ & $\begin{array}{l}\text { El gerente del deporte orienta la } \\
\text { gestión organizativa soportado } \\
\text { en los procesos de } \\
\text { planificación, organización, } \\
\text { dirección y control. }\end{array}$ & & $\begin{array}{l}\text { No se evidencia una formación } \\
\text { real sobre los procesos } \\
\text { administrativos que orienten la } \\
\text { conducción idónea de los } \\
\text { destinos del deporte. }\end{array}$ \\
\hline Acosta (2005) & $\begin{array}{c}\text { CAPACIDADES } \\
\text { INTERPERSONALES }\end{array}$ & $\begin{array}{l}\text { Se debe poseer conocimientos } \\
\text { acerca de los niveles de trabajo, } \\
\text { las habilidades y capacidad de } \\
\text { los empleados, las funciones y } \\
\text { la motivación respecto a los } \\
\text { objetivos de la organización. }\end{array}$ & & $\begin{array}{l}\text { Existe en estos cargos } \\
\text { personas con bajo nivel cultural } \\
\text { que se traduce en un manejo } \\
\text { inadecuado de las relaciones } \\
\text { ente pares y colaboradores. }\end{array}$ \\
\hline $\begin{array}{l}\text { Vargas (2011) y } \\
\text { Campos (2007) }\end{array}$ & $\begin{array}{l}\text { FORMACIÓN Y } \\
\text { CAPACITACIÓN }\end{array}$ & $\begin{array}{l}\text { Un gerente del deporte debe } \\
\text { tener formación académica y } \\
\text { capacitación en competencias } \\
\text { de dirección, debiendo } \\
\text { seleccionarse los más } \\
\text { calificados para conducir } \\
\text { exitosamente el sistema } \\
\text { deportivo. }\end{array}$ & & $\begin{array}{l}\text { No se tiene la formación } \\
\text { requerida para el cargo, } \\
\text { además que buena parte de la } \\
\text { dirigencia ecuatoriana no posee } \\
\text { nivel universitario, acarreando } \\
\text { un limitado conocimiento en } \\
\text { materia gerencial. }\end{array}$ \\
\hline $\begin{array}{l}\text { Aguilar y Guerra } \\
\text { (2014) }\end{array}$ & $\begin{array}{l}\text { EXPERIENCIA EN } \\
\text { EL DEPORTE }\end{array}$ & $\begin{array}{l}\text { Se debe tener experiencia } \\
\text { lograda mediante su práctica en } \\
\text { escenarios deportivos, aspecto } \\
\text { que ayudaría a tener un } \\
\text { conocimiento anticipado de las } \\
\text { circunstancias que rodean a } \\
\text { este sector. }\end{array}$ & $\begin{array}{l}\text { Es práctica común el ejercicio } \\
\text { de una gerencia basada en la } \\
\text { experiencia que se tiene en el } \\
\text { deporte. }\end{array}$ & \\
\hline
\end{tabular}

Fuente: Elaboración propia basada en los aportes teóricos y los hallazgos encontrados en la investigación. 
deporte" como aporte teórico. Una experiencia que ayudaría a entender el significado de la constancia, la disciplina, la dedicación y el sacrificio que dedica un atleta a su vida deportiva; factores que facilitarían la toma de decisiones y la búsqueda de soluciones a los problemas que enfrenta la gerencia del deporte tanto del alto rendimiento como de las ligas barriales.

En lo atinente a la categoría Características de la Cultura Organizacional (Tabla 5), se detectó que la realidad se exhibe de manera contradictoria al contrastarla con los aportes "Alineamiento de acciones" e "Imperativos gerenciales", como resultante de una cultura organizacional que se caracteriza por un manejo discrecional de las decisiones que se toman en las altas esferas del poder político deportivo, afectando aguas abajo la coordinación de esfuerzos en función de los intereses comunes del deporte de alto rendimiento y ligas barriales.

Aun así, se valoró una cultura organizacional que funciona con una simbología común, evidencia que coincide con el aporte "Sistemas simbólicos". Así pues, los sistemas simbólicos en el deporte

Tabla 5.

Coincidencias y contradicciones en características de la cultura organizacional.

\begin{tabular}{|c|c|c|c|c|}
\hline \multirow{2}{*}{ AUTORES } & \multirow{2}{*}{ APORTES } & \multirow{2}{*}{ ESTADO DEL ARTE } & \multicolumn{2}{|c|}{ REALIDAD EMPÍRICA } \\
\hline & & & COINCIDENCIAS & CONTRADICCIONES \\
\hline $\begin{array}{c}\text { Gómez (2017); Geertz } \\
\text { (1987) y Schneider } \\
\text { (1980) }\end{array}$ & $\begin{array}{l}\text { SISTEMAS } \\
\text { SIMBÓLICOS }\end{array}$ & $\begin{array}{l}\text { El comportamiento social y el } \\
\text { lenguaje entre pares, } \\
\text { colaboradores y relacionados } \\
\text { son códigos que se relacionan } \\
\text { entre sí y en su esencia } \\
\text { responden a un sistema de } \\
\text { símbolos culturales basados en } \\
\text { normas y reglas que deben } \\
\text { cumplirse. }\end{array}$ & $\begin{array}{l}\text { Las organizaciones del deporte } \\
\text { funcionan con códigos propios } \\
\text { bien definidos que los } \\
\text { diferencian de otras } \\
\text { organizaciones. Es un lenguaje } \\
\text { común entendido por todos y } \\
\text { además existe una Ley del } \\
\text { Deporte que regula las } \\
\text { responsabilidades, funciones y } \\
\text { tareas. }\end{array}$ & \\
\hline $\begin{array}{l}\text { Levy-Strauss (1985) y } \\
\text { Goodenough (1981) }\end{array}$ & $\begin{array}{c}\text { ALINEAMIENTO DE } \\
\text { ACCIONES }\end{array}$ & $\begin{array}{l}\text { Un grupo de personas } \\
\text { organizan sus experiencias en } \\
\text { torno a una realidad concreta a } \\
\text { través de sus percepciones, } \\
\text { comprendiendo e interpretando } \\
\text { las interacciones cotidianas que } \\
\text { orientan el comportamiento de } \\
\text { sus miembros. }\end{array}$ & & $\begin{array}{l}\text { Las autoridades deportivas } \\
\text { exigen resultados sin pasar por } \\
\text { el tamiz de una evaluación } \\
\text { previa y no realizan análisis en } \\
\text { conjunto con las organizaciones } \\
\text { del deporte para articular } \\
\text { expectativas y acciones que se } \\
\text { desean cumplir. }\end{array}$ \\
\hline Kotter (2007) & $\begin{array}{l}\text { IMPERATIVOS } \\
\text { GERENCIALES }\end{array}$ & $\begin{array}{l}\text { Una cultura organizacional debe } \\
\text { ser conducida por un gerente } \\
\text { que además de planificar, } \\
\text { estructurar y sistematizar las } \\
\text { complejidades organizativas, } \\
\text { tenga la capacidad para inspirar } \\
\text { a otros. }\end{array}$ & & \begin{tabular}{|l} 
La presencia de espacios de \\
poder político en distintas \\
instancias inciden de manera \\
importante en las decisiones \\
que se toman; circunstancias \\
que atentan contra el necesario \\
respaldo para reconducir \\
acciones que permitan el \\
posicionamiento deportivo a \\
escala internacional del alto \\
rendimiento y la atención \\
equitativa a todas las ligas \\
barriales.
\end{tabular} \\
\hline
\end{tabular}

Fuente: Elaboración propia basada en los aportes teóricos y los hallazgos encontrados en la investigación. 
se caracterizan por el uso de códigos propios para comunicarse entre pares, colaboradores y relacionados, y adicionalmente las funciones están soportadas en la Ley del Deporte.

Con respecto a la categoría Concepción de la Cultura Gerencial (Tabla 6), se reveló una actuación gerencial que a todas luces se contradice con el total de aportes seleccionados en este segmento, como son "Motivación inspiradora", "Esfuerzo vectorial", "Esfuerzo cooperativo" y "Perspectiva gerencia".

A tal efecto, es un comportamiento que denota falta de herramientas gerenciales para manejar con habilidad a las personas, inyectando dinamismo, sumando voluntades y convenciendo a la gente para alcanzar los objetivos y metas planteadas. En suma, son debilidades que no permiten en un gerente del deporte

Tabla 6.

Coincidencias y contradicciones en concepción de la cultura gerencial.

\begin{tabular}{|c|c|c|c|c|}
\hline \multirow{2}{*}{ AUTORES } & \multirow{2}{*}{ APORTES } & \multirow{2}{*}{ ESTADO DEL ARTE } & \multicolumn{2}{|c|}{ REALIDAD EMPÍRICA } \\
\hline & & & COINCIDENCIAS & CONTRADICCIONES \\
\hline Gómez (2017) & $\begin{array}{l}\text { MOTIVACIÓN } \\
\text { INSPIRADORA }\end{array}$ & $\begin{array}{l}\text { Se inspira al equipo en la } \\
\text { búsqueda de valoración hacia el } \\
\text { trabajo, la cohesión grupal, la } \\
\text { creación de procesos de } \\
\text { comunicación por razones } \\
\text { técnicas o por motivos afectivos } \\
\text { y emocionales, la instauración } \\
\text { de un sistema de valores y } \\
\text { creencias compartidas. }\end{array}$ & & $\begin{array}{l}\text { Se adolece de gerentes del } \\
\text { deporte con las herramientas } \\
\text { necesarias vinculadas a } \\
\text { competencias blandas } \\
\text { requeridas para relacionarse } \\
\text { con otros en el interés de } \\
\text { manejar desacuerdos y detectar } \\
\text { oportunidades de mejora. }\end{array}$ \\
\hline $\begin{array}{c}\text { Etking y Schvarstein } \\
\text { (1992) }\end{array}$ & $\begin{array}{l}\text { ESFUERZO } \\
\text { VECTORIAL }\end{array}$ & $\begin{array}{l}\text { La cultura gerencial condiciona } \\
\text { los modos de pensar, crecer, } \\
\text { hacer y cumplir las funciones y } \\
\text { tareas gerenciales dentro de } \\
\text { una organización. }\end{array}$ & & $\begin{array}{l}\text { El comportamiento gerencial se } \\
\text { circunscribe a cumplir con un } \\
\text { Plan Operativo Anual por } \\
\text { exigencias presupuestarias del } \\
\text { Ministerio del Deporte, ente } \\
\text { administrativo que no funciona } \\
\text { con planes estratégicos, con el } \\
\text { agravante que no son los más } \\
\text { capacitados, mostrando poco } \\
\text { conocimiento sobre el deporte. }\end{array}$ \\
\hline $\begin{array}{c}\text { Etking y Schvarstein } \\
\text { (1992) }\end{array}$ & $\begin{array}{c}\text { ESFUERZO } \\
\text { COOPERATIVO }\end{array}$ & $\begin{array}{l}\text { La cultura gerencial favorece la } \\
\text { formas de interacción social } \\
\text { que son comunicadas y } \\
\text { difundidas, y a su vez } \\
\text { compartidas por los grupos. }\end{array}$ & & $\begin{array}{l}\text { Existe una alta dirigencia que en } \\
\text { sí no tiene una clara visión } \\
\text { deportiva. En este sentido, si no } \\
\text { hay direccionalidad para } \\
\text { controlar la gestión de un } \\
\text { Ministerio, menos aún en las } \\
\text { demás esferas de la estructura } \\
\text { deporta. }\end{array}$ \\
\hline Granell (1997) & $\begin{array}{l}\text { PERSPECTIVA } \\
\text { GERENCIAL }\end{array}$ & $\begin{array}{l}\text { La cultura gerencial está } \\
\text { focalizada en la incubación de } \\
\text { cambios y transformaciones } \\
\text { que sean provechosas y } \\
\text { pertinentes para el éxito } \\
\text { organizativo, sin perder de vista } \\
\text { aquellas transformaciones que } \\
\text { se produzcan en la aldea global. }\end{array}$ & & $\begin{array}{l}\text { No hay un pensamiento } \\
\text { estratégico que oriente las } \\
\text { acciones futuras. Se debiera } \\
\text { observar las mejores prácticas } \\
\text { gerenciales en equipos } \\
\text { deportivos de clase mundial y } \\
\text { de aquellos países de la región } \\
\text { como Perú, Venezuela y } \\
\text { Colombia, que aventajan a } \\
\text { Ecuador en el tema gerencial. }\end{array}$ \\
\hline
\end{tabular}

Fuente: Elaboración propia basada en los aportes teóricos y los hallazgos encontrados en la investigación. 
observar las mejores prácticas de otros países en materia gerencial, los cuales han alcanzado un posicionamiento importante en el ámbito deportivo internacional. Son aspectos que sin duda no contribuyen a concebir una cultura gerencial que pueda lograr los cambios y transformaciones que demanda en el deporte de alto rendimiento y barrial en Ecuador.

A la luz de estas comparaciones, se puede evidenciar que existen más contradicciones que coincidencias entre lo que plantea la teoría y lo que acontece en la realidad. Una brecha que afecta de manera importante los resultados esperados, no sólo por las personas que están involucradas con el deporte, sino también por una sociedad ecuatoriana que demanda efectividad de la gerencia del deporte, cuyo foco está en las ligas barriales donde se favorece a grandes sectores de la sociedad y nacen los deportistas del futuro, y en el alto rendimiento, donde se alcanzan los más elevados estándares de calidad y excelencia competitiva.

\section{Conclusiones}

La investigación realizada se soportó en un enfoque metodológico alineado con las orientaciones que brindan Strauss y Corbin (2002), cuya pertinencia estuvo confirmada por la validez y confiabilidad de los resultados que se generaron mediante un proceso que aplicó el método comparativo constante en la búsqueda de patrones, regularidades, inconsistencias, incoherencias y discontinuidades de datos provenientes de la realidad estudiada de 4 entrevistados con responsabilidades gerenciales, con la condición de ser líderes de opinión con rango y fuerza para suministrar información relevante $\mathrm{y}$ atinente a través de entrevistas en profundidad con respecto a la cultura gerencial del deporte en Ecuador.

A tal efecto, los hallazgos que emergieron en este estudio confirman que las organizaciones del alto rendimiento y del deporte barrial precisan una cultura gerencial sólida que delimite su comportamiento. Una cultura gerencial que se sustente por una parte en el conocimiento logrado tanto en las universidades como en los procesos de capacitación gerencial y articulado con la experiencia lograda en años dentro del deporte; y por otra, con aquellos aspectos intangibles y subjetivos que trastocan la dimensión humana. Por consiguiente, un desempeño exitoso dependerá de la combinación de este conjunto de factores que condicionan la cultura gerencial.

Así pues, estos factores adecuadamente alineados bajo un destino compartido, donde exista continuidad administrativa en las altas esferas del deporte para responder a proyectos comunes, contribuirían al afianzamiento de una sólida plataforma deportiva que permita ir edificando las bases para encontrar caminos hacia una ajustada cultura gerencial con un sistema de creencias, actitudes, valores, rutinas $\mathrm{y}$ hábitos que ayuden a cambiar el estado actual de cosas en el interés común de transformar definitivamente al deporte ecuatoriano y lo conduzcan hacia el éxito 
en el contexto local, nacional e internacional. En definitiva, las aportaciones que se derivan de este estudio, servirán de plataforma para la consecución de investigaciones de igual o similar naturaleza en otros segmentos del deporte.

\section{Referencias}

Acosta, R. (2005). Gestión y administración de organizaciones deportivas. Barcelona: Editorial Paidotribo.

Aguilar, P. y Guerra, W. (2015). Gerencia del deporte de alto rendimiento en Venezuela. Actualidad Contable FACES, 18 (31), 5-29.

Aguilar, P. (2014). Gerencia del deporte de alto rendimiento en Venezuela: hacia una implantación efectiva de los procesos administrativos. Saarbrücken: Editorial Publicia.

Arias, F. (2017). Economía y deporte. Caracas: Editorial Episteme.

Arias, F. (2016). El Problema de Investigación: Introducción a la Metodología Científica. Caracas: Editorial Episteme.

Calabuig, F., Gómez, A., y Liceras, J. (2010). El Perfil del Gerente Deportivo. International Journal of Sports \& Management, 9, 24-47.

Campos, A. (2007). Los profesionales de la organización y gestión de actividad física $y$ deporte en las instalaciones deportivas $y$ entidades. Madrid: Universidad Politécnica de Madrid.

Cazorla, L. (1979). Deporte y Estado. Barcelona: Editorial Labor.

Cerda, H. (1991). Los elementos de la investigación. Bogotá: El Búho.
Etking, J. y Schvarstein, L. (1992). La Identidad de las Organizaciones. Buenos Aires: Paidós.

Geertz, C. (1987). La interpretación de las culturas. México: Editorial Gedisa.

Gómez, S. (2017). Cultura gerencial en las organizaciones del deporte: Valores, creencias, prácticas, herramientas, métodos y procedimientos en organizaciones del deporte. Saarbrücken: Editorial Académica Española.

Goodenough, W. H. (1981). Culture, language and society. Reading, Mass: Addison-Wesley.

Granell, E. (1997). Exito Gerencial y Cultura: Ritos y oportunidades para Venezuela. Caracas: Instituto de Estudios Superiores de Administración-IESA.

Kotter, J. (2007). Al frente del cambio. México: Editorial Empresa Activa.

Levy-Strauss, C. (1985). Las estructuras elementales del parentesco. México: Planeta.

Martínez, M. (2009). Ciencia y arte en la metodología cualitativa. México: Trillas.

Peiró, J. Ramos, J. y Martínez-Tur, V. (1995). La Gestión de las Organizaciones Deportivas y su Problemática. En: J. Peiró y J. Ramos (Directores), Gestión de Instalaciones Deportivas Una Perspectiva Psicosocial. Pp. 13-26. Valencia.

Rusque, A. (2007). De la diversidad a la investigación cualitativa. Caracas: Vadel.

Schneider, D. (1980). American Kinship, a Cultural Account. Chicago: The Universisity of Chicago.

Strauss, A. y Corbin, J. (2002). Bases de la investigación cualitativa: técnicas y procedimientos para desarrollar la teoría fundamentada. Medellín: Universidad de Antioquia. 
Vargas, C. (2011). Enfoque de gestión pública y deporte. En: Asociación Latinoamericana de Estudios Socioculturales del Deporte 2011: Actividad físico-corporal, deporte, sociedad y crítica social. (pp. 400-412).

Maracay: Centro de investigación EDUFISADRED.

Villegas, C. (2012). El Deporte desde una Visión Transcompleja. Revista Cientifica Dimensión Deportiva, 3, 9-16. 